\title{
A Retrospective Study of Poultry Diseases Diagnosed at the Veterinary Laboratory, Pokhara, Nepal
}

\author{
B. Regmi ${ }^{1, *}$, I. Dhakal ${ }^{2}$, M. K. Shah ${ }^{3}$, K. R. Pande ${ }^{1}$ \\ ${ }^{1}$ Veterinary Laboratory, Pokhara, Nepal. \\ ${ }^{2}$ Army Equine Breeding Center, Chitwan, Nepal. \\ ${ }^{3}$ Department of Surgery \& Pharmacology, AFU, Chitwan, Nepal.
}

How to cite this paper: B. Regmi, I Dhakal, M. K. Shah, K. R. Pande. (2020) A Retrospective Study of Poultry Diseases Diagnosed at the Veterinary Laboratory, Pokhara, Nepal. International Journal of the Science of Food and Agriculture, 4(4), 398-402.

DOI: $10.26855 /$ ijfsa.2020.12.006

Received: September 16, 2020

Accepted: October 12, 2020

Published: October 28, 2020

*Corresponding author: B. Regmi, Veterinary Laboratory, Pokhara, Nepal. Email: regmibharat2008@gmail.com

\begin{abstract}
A retrospective study of clinical case records of poultry diseases presented to the pathology unit of Veterinary Laboratory (VL), Pokhara was conducted for duration of one year (March 2018 to February 2019). The basis of diagnosis were necropsies, rapid diagnostic test (RDT), plate agglutination test (PAT), microscopic examination, ELISA and PCR. The diagnosed diseases were bacterial including colibacilosis, omphalitis, salmonellosis, chronic respiratory disease (CRD) and complicated chronic respiratory disease (CCRD); parasitic including coccidiosis, tapeworm and ascariasis; viral including new castle disease (ND), infectious bronchitis (IB), infectious bursal disease (IBD), Marek's disease (MD) and avian influenza (AI) and non- infectious diseases/disorders including immunosuppression, mycotoxicity (MT), enteritis, pneumonitis, ascites, dehydration, nephritis, hepatitis, gout, sudden death syndrome (SDS), paralysis, fatty liver syndrome (FLS), hydro pericardial syndrome (HPS) and necrotic enteritis (NE). Out of total 5,037 cases recorded, bacterial diseases accounted for $41.16 \%$, followed by parasitic (22.67\%), non-infectious (19.93\%) and 16.24\% viral. Colibacilosis (14.49\%), IBD (12.67\%) and coccidiosis (22.63\%) were the most prevalent diseases in their respective category. The highest prevalence of overall diseases were found in the spring season (27.24\%), followed by winter (26.70\%), summer (23.94\%) and autumn (22.12\%). Out of 2,024 caecal scrapings taken from the chicken of more than two weeks of age and tasted for caecal coccidian oocyst, 583 (28.8\%) were found positive. The prevalence of coccidiosis was found more in humid spring season (28.3\%), followed by rainy (26.9\%), autumn (24.4\%) and winter (20.4\%). On the rapid PAT of morbid chickens, $46.84 \%$ Salmonella and 56.51\% Mycoplasma were found sero-positive. Similarly, 62.97\% IBD, 42.02\% ND and 9.5\% AI were positive for their specific antigens on RDT. Majority of the cases were found related to poor management and biosecurity measures of farm. Therefore, knowledge on seasonal occurrence of different diseases, their preventive measures as well as accurate diagnosis and treatment are the key factors for successful poultry farming.
\end{abstract}

\section{Keywords}

Retrospective study, poultry disease, Veterinary Laboratory, diagnosis

\section{Introduction}

Poultry production in Nepal is an important component of livestock sector which contributes $3 \%-4 \%$ in total gross domestic product (GDP) and $8 \%$ in agricultural gross domestic product [1, 2]. Nepal is self-sufficient for egg production and moving towards the poultry meat too. Many districts are becoming self-reliant for both of poultry products. 
The growth rate of commercial sector is around $17 \%-18 \%$ annually and its contribution to overall GDP is also encouraging and increasing [1]. There is an investment of NRs 80 billion in this sector which has provided direct employment of 1.5 lakh people [3]. With the intensification and industrialization of poultry sector, different diseases are increasing and causing huge economic losses, some of which are of high zoonotic potential like Avian Influenza (AI).

The western regional districts of Nepal contribute $15.89 \%$ of total chicken population of country [1]. Kaski is one of the district with large poultry population where $9.65 \%$ are backyard, $21.86 \%$ are commercial chickens both of which contributes $18.94 \%$ among the six main districts for poultry commercialization (Kaski, Chitwan, Biratnagar, Kathman$\mathrm{du}$, Bhaktapur and Lalitpur) [1]. Diseases are major constraints in the development of poultry industry, thus causing a huge loss to the farmers. Many diseases have seasonal occurrence $[4,5]$. Therefore, this study was aimed to find out the prevalence of different poultry disease and their spatial and temporal occurrence through the study of cases recorded at the Veterinary laboratory, Pokhara.

\section{Material and Methods}

\subsection{Study Area}

Pokhara and its periphery area has a humid subtropical climate with four main seasons; dry winter (December-February), hot and dry spring (March-May), very hot and humid rainy (June-August) and moderate autumn (September-November).

\subsection{Methodology}

All the cases brought to the Veterinary Laboratory (VL) were diagnosed by veterinarian mainly on the basis of history, post mortem examination (PME), microscopic examination, rapid plate agglutination test (PAT), rapid diagnostic test (RDT) for antigen detection, enzyme linked immunosorbent assay (ELISA) and polymerase chain reaction (PCR). Coccidian parasites were confirmed through caecal scrapings and their microscopic examination by viewing the oocytes. Sero-prevalence of some bacterial diseases like Salmonella and Mycoplasma gallisepticum (MG) were performed for the morbid chicken by PAT using commercial Salmonella and MG antigens to determine the presence of Salmonella and MG specific antibodies. RDT was used for different viral diseases like new castle disease (ND), infectious bursal disease (IBD), infectious bronchitis (IB) and AI. Sometimes, bacterial culture and biochemical test were utilized for the diagnosis of some bacterial diseases like salmonellosis, colibacilosis and pasturellosis. On the basis of cultural characteristics and biochemical test the causative organisms were identified.

\subsection{Data collection and Analysis}

The one year (March 2018 to Feb 2019) poultry disease diagnostic records on 5,037 cases (necropsies and other associated tests) maintained in VL were subjected to a retrospective epidemiological analysis for the prevalence of different poultry diseases. Recorded cases were counted and grouped under various categories such as: breed, disease, season and districts. Data were analyzed using Microsoft excel-2013 and the results of the analysis were determined and expressed in the percentage distribution in chart forms.

\section{Results and Discussion}

Out of the total cases presented, $80 \%$ were broilers, $12 \%$ layers and remaining $8 \%$ were local, giriraj, fighter and turkey. Most of the cases (85\%) recorded were from small farmers $(<2,000$ birds), followed by $10 \%$ medium farmers (2,000-5,000 birds) and remaining 5\% large farmers (> 5,000 birds). Majority of the cases were from Kaski (81\%) followed by Tanahun (9.7\%), Syangja (5.7\%) and other districts (3.6\%) as shown in Figure 1A.

The most frequently diagnosed diseases were coccidiosis $(1,140)$, non- infectious disease/disorders $(1,004)$, colibacillosis (730), followed by IBD (638), chronic respiratory disease (CRD) (575), salmonellosis (431), complicated chronic respiratory disease (CCRD) (178), omphalitis (159), ND (147) and others (IB, MD, AI, tapeworm and ascariasis) as shown in Figure 1C and Figure 1D, which is in contrary to the findings of Gautam et al. [5] who reported colibacilosis as the most frequently investigated diseases followed by coccidiosis, mycotoxicity (MT), IBD, CRD, salmonellosis, ND and Marek's disease (MD). It was found that most of the cases were flowed during spring season followed by winter (Figure 1B).The main bacterial disease, omphalitis and colibacilosis is caused by E. coli which causes heavy economic losses to the poultry industry by its association with various disease conditions, either as primary pathogen or as a secondary pathogen [6]. It is particularly evident in the poultry farms of developing countries, which due to poor bio-security systems are extremely prone to this disease [7]. The overall prevalence of caecal coccidiosis (28.8\%) as shown in Figure 2E, is lower than 39.58\% as reported by Ebrahimi-Nik et al. [7] and 31.8\% reported by Lawal et al. [8]. The main parasitic disease, coccidiosis is due to poor management practices in the deep litter system encouraging Eimeria oocysts to build-up. The prevalence of Salmonella and Mycoplasma infection (46.84\% and $56.51 \%$ respectively) on PAT (Figure 2A) is higher than the findings of Sikder et al. [9], who had recorded $23.46 \%$ salmonellosis and $46.88 \%$ mycoplasmosis. This higher value indicates the poor management and biosecurity measures of farming system. Salmonellosis was found the most prevailing disease in winter season where as Mycoplasmosis in rainy season (Figure 2B). The prevalence of IBD (62.97\%) on RDT is similar to the finding of Lawal et al. [10] who have reported the prevalence 
of 63.5\%. This high prevalence may be due to the sample taken from only suspected chickens on PME. But the overall IBD prevalence (12.67\%) among several diseases shows that it is prevailing in endemic condition in Kaski district and its periphery area.

Similarly, the prevalence of $48.02 \%$ was found for ND on RDT from the suspected chicken carcass (Figure 2C) which is higher than the findings of Rahman et al. [11], who reported about one third sample positive. A high prevalence of IBD (33\%) and ND (39.7\%) was obtained during the rainy season as compared to dry winter season (Figure 2D). This is in consistent with the report of Lawal et al. [10], who also reported high prevalence of IBD 84.4\% (rainy season) and $40.3 \%$ (dry season). The prevalence of AI was found $9.46 \%$, in which high prevalence was in spring $(43.33 \%)$ and winter season (40\%) which may be due to the high susceptibility of virus in cool and moist climatic conditions.
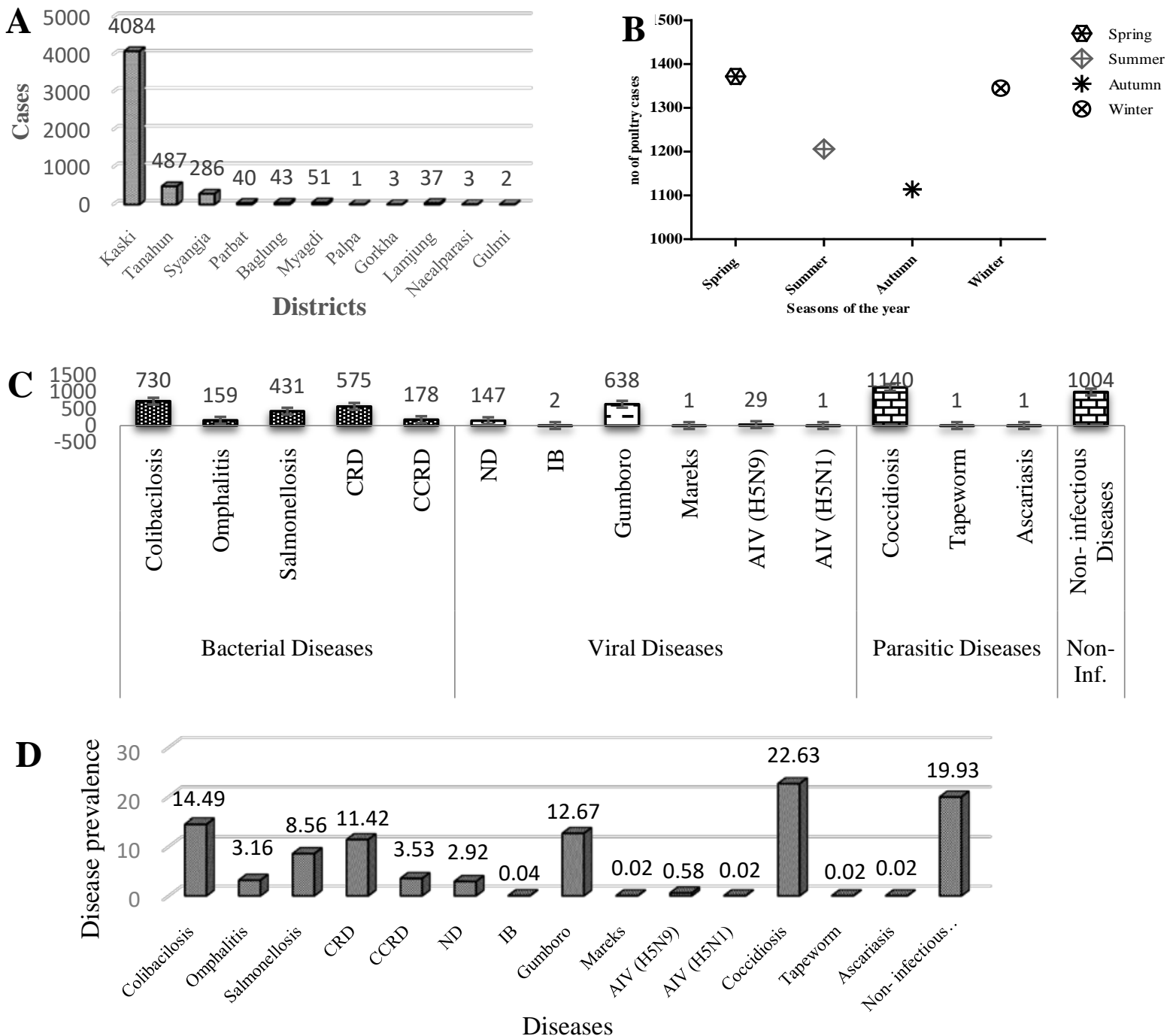

Figure 1. Diagram showing, A: Poultry case flow pattern from different districts, showing majority of cases $(4,084)$ from Kaski district; B: Seasonal case flow pattern of poultry diseases, showing lowest case flow pattern during autumn. The overall disease case flow was found in the spring season (27.24\%), followed by winter (26.70\%), summer (23.94\%) and autumn (22.12\%); C: Occurrence of different poultry diseases; showing highest prevalence of bacterial (41.16\%), followed by parasitic (22.67\%), non-infectious (19.93\%) and viral (16.24\%) cases; D: Prevalence of different poultry diseases; colibacillosis $(14.49 \%)$, CRD $(11.42 \%)$, coccidiosis $(22.63 \%)$ and Gumboro $(12.67 \%)$ were major diseases in their respective disease categories of bacterial, parasitic and viral. 
A

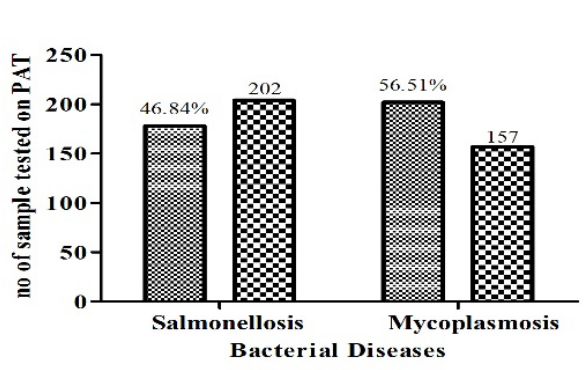

C

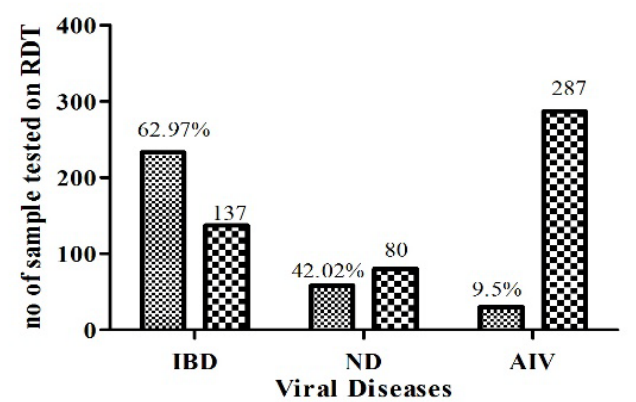

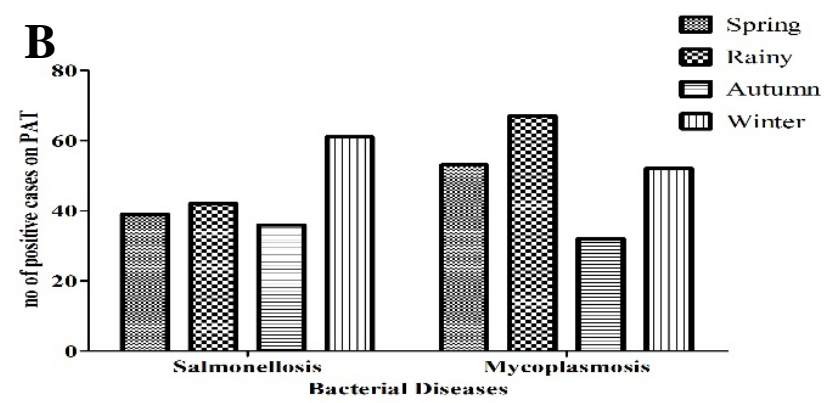

D
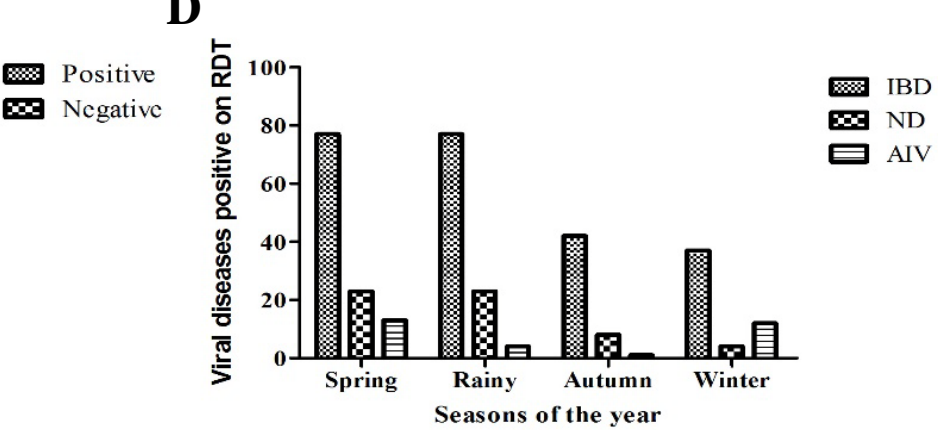

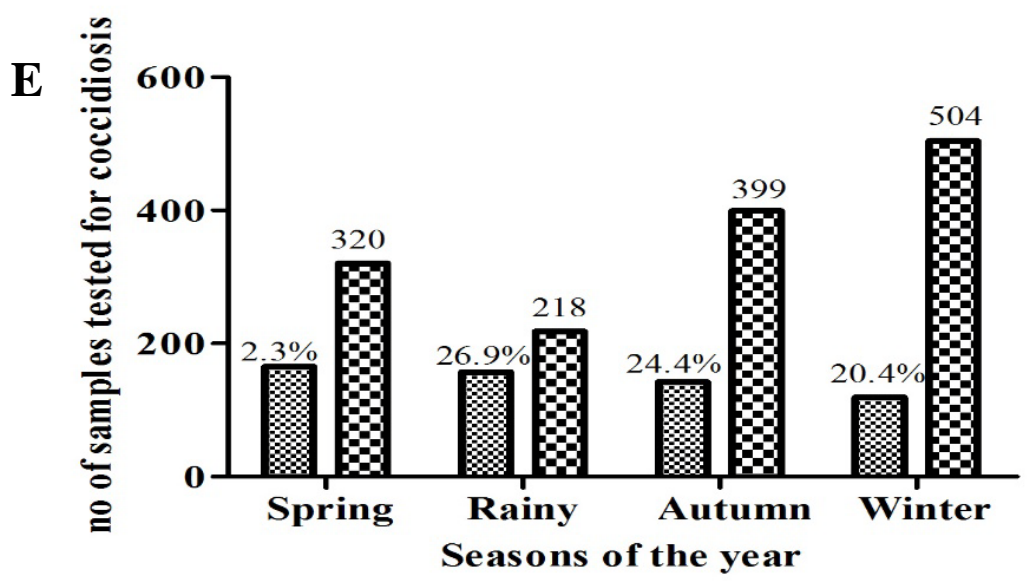

\& Positive

ax Negative

Figure 2. Diagram showing, A: Sero-prevalence of Salmonellosis \& Mycoplasmosis based on rapid PAT, showing the prevalence of Salmonella and Mycoplasma infection were $46.84 \%$ and $56.51 \%$ respectively; B: Seasonal occurrence of Salmonellosis \& Mycoplasmosis, showing the highest prevalence of Salmonella in winter season 61 (34.27\%) and Mycoplasma in rainy season 67 (32.84\%); C: Different viral diseases positive on RDT; the prevalence of IBD, ND and AI was found 62.97\%, 42.02\% and 9.5\% respectively on the RDT; D: Seasonal variation of IBD, ND and AI diseases, showing the higher cases of IBD, ND in spring and rainy season whereas AI in spring \& winter; E: Seasonal variation of caecal coccidiosis on microscopic examination. Out of the 2,024 caecal scrapings taken from the chicken of more than two weeks of age and tasted for caecal coccidian oocyst, $583(\mathbf{2 8 . 8} \%)$ were found positive. The prevalence of coccidiosis was found more in humid spring season (28.3\%), followed by rainy (26.9\%), autumn $(24.4 \%)$ and winter $(20.4 \%)$.

\section{Conclusion}

On the basis of history, PME, microscopic examination, rapid PAT and RDT, various poultry diseases can be diagnosed but some may require histopathology, ELISA and PCR for the precise diagnosis. Rapid tests are very easy, quick, less laborious and non-expensive for the detection and differentiation of different diseases.

Most of the problems recorded were found correlated with the poor management and biosecurity measures of farm. Therefore, the farmers must be conscious about seasonal occurrence of common poultry diseases, their timely vaccination, good farm management and overall biosecurity measures. 


\section{Conflict of Interest}

The authors declare that there is no conflict of interest.

\section{References}

[1] FAO. (2014). Poultry Sector Nepal. Retrived September 9, 2020, fromhttp://www.fao.org/3/a-i3964e.pdf.

[2] Kattel, P. (2016). Socio-Economic Importance of Indigenous Poultry in Nepal. Poultry, Fish Wildl Sci, 3(1). DOI: 10.4172/2375-446X.1000153.

[3] Singh, S. (2018). Overview of Nepalese Poultry Industry. Retrived September 9, 2020, from http://www.vcn.gov.np/uploads/files/Overview\%20of\%20Nepalese\%20Poultry\%20Industry_Dr_\%20Subir.pdf.

[4] Regmi, B., Dhakal, I., Chetri, D., and Shah, M. K. (2020). Clinical Prevalence of Diseases and Disorders in Buffaloes at the Veterinary Teaching Hospital, Agriculture and Forestry University (AFU), Nepal. Int J Food Sci Agric, 4(2): 203-210. DOI: 10.26855/ijfsa.2020.06.012.

[5] Gautam, G., Devkota, B., and Thapaliya, S. (2017). Recent case flow pattern in Veterinary Teaching Hospital of Agriculture and Forestry University, Chitwan, Nepal. J Agric For Univ, vol. 1. Retrived September 9, 2020, from http://afu.edu.np/sites/default/files/Recent_case_flow_pattern_in_Veterinary_Teaching_Hospital_of_Agriculture_and_Forestry _University_Chitwan_Nepal_G._Gautam.pdf.

[6] Lutful Kabir, S. M. (2010). Avian colibacillosis and salmonellosis: a closer look at epidemiology, pathogenesis, diagnosis, control and public health concerns. Int J Environ Res Public Health, 7(1): 89-114. DOI: 10.3390/ijerph7010089.

[7] Ebrahimi-Nik, H., Bassami, M. R., Mohri, M., Rad, M., and Khan, M. I. (2018). Bacterial ghost of avian pathogenic E. coli (APEC) serotype O78:K80 as a homologous vaccine against avian colibacillosis. PLoS One, 13(3): p. e0194888. DOI: 10.1371/journal.pone.0194888.

[8] Lawal, J. R., Jajere, S. M., Ibrahim, U. I., Geidam, Y. A., Gulani, I. A., Musa, G., and Ibekwe, B. U. (2016). Prevalence of coccidiosis among village and exotic breed of chickens in Maiduguri, Nigeria. Vet world, 9(6): 653-659. DOI: 10.14202/vetworld.2016.653-659.

[9] Sikder, A., Islam, M., Rahman, M., and Rahman, M. (2005). Seroprevalence of Salmonella and Mycoplasma gallisepticum Infection in the Six Model Breeder Poultry Farms at Patuakhali District in Bangladesh. Int J Poult Sci, 4(11): 905-910. DOI: 10.3923/ijps.2005.905.910.

[10] J. R. Lawal, S. M. Jajere, A. M. Bello, M. Mustapha, Y. Wakil, J. J. Ndahi, F. B. Mustapha, B. T. Paul, I. A. Gulani, U. I. Ibrahim, Y. A. Geidam, A. G. Ambali, and I. Waziri. (2014). Prevalence of infectious Bursal disease (Gumboro) Antibodies in village Chickens in Gombe State, Northeastern Nigeria. Int J Poult Sci, 13(12): 703-708. DOI: 10.3923/ijps.2014.703.708.

[11] Rahman, M. S., Rabbani, M. G., Uddin, Chakrabartty, M. J., A., and Her, M. (2012). Prevalence of Avian Influenza and Newcastle Disease Viruses in poultry in selected areas of Bangladesh using rapid antigen detection kit. Arch Clin Microbiol, 3(1). DOI: $10.3823 / 248$. 